\title{
Mecânica Clássica - Referenciais Inerciais e Energia
}

\author{
M.C.Bertin ${ }^{1}$ and Mario Cezar Bertin ${ }^{2}$ \\ ${ }^{1}$ Universidade Federal da Bahia \\ ${ }^{2}$ Instituto de Física, Universidade Federal da Bahia
}

September 24, 2020

Rich media available at https://youtu.be/LNdpTB3w70Y

\section{Os quatro primeiros postulados}

Vamos apresentar novamente os postulados que introduzimos até aqui:

Postulado 1: A posição de uma partícula é representada por um ponto em R 3 .

Postulado 2: A distância entre duas partículas é representada pela distância euclidiana em R 3

Postulado 3: O movimento de uma partícula é representado por uma curva suave em R 3 .

Postulado 4: O tempo é representado pelo parâmetro das curvas em R 3 com as propriedades:

Homogeneidade, Invariância do intervalo de tempo entre observadores, Crescimento monotônico.

Com estes postulados, fomos capazes de introduzir observáveis intrínsecos, que são definidores da partícula, como a massa e a carga elétrica. Ainda, vimos que existem outros observáveis, os observáveis extrínsecos, que possuem relação com a partícula. Os postulados 3 e 4 são, para todos os efeitos, os postulados que introduzem o fenômeno do movimento, então, podemos agora dizer que os observáveis extrínsecos são responsáveis por definir estados de movimento, ou seja, possuem relação com a partícula mas pertencem, em última instância, à forma como esta se move no espaço.

Os observáveis que vimos até agora são representados por campos vetoriais, ou seja, são vetores cujas normas são invariantes por rotações. O postulado 2 introduz o observável posição de uma partícula, representado pelo vetor posição $\mathbf{x}$, mas o movimento ocorre através de curvas suaves no espaço, como proposto pelo postulado 3. Portanto, a posição, assim como outros observáveis, dependem do parâmetro temporal, aquele do quarto postulado. A suavidade das curvas em $\mathbb{R}^{3}$ induz a existência de outros dois observáveis, a velocidade e a aceleração. Estes observáveis são campos vetoriais, dependem portanto do ponto sobre a trajetória e esta, em razão da curva que representa o movimento, depende do tempo.

Contudo, ainda não temos uma regra que nos diga a forma pela qual uma partícula se desloca sobre uma trajetória. Ou seja, dado um conjunto definido de observáveis relacionados a uma partícula, como saber sob qual curva ela se movimenta? Aqui, trabalharemos com a seguinte ideia: à medida que a partícula percorre uma trajetória, o movimento deixa invariante um conjunto específico de observáveis físicos, denominados invariantes dinâmicos. Assim, os invariantes dinâmicos serão quantidades físicas que não mudam seu valor na medida que o tempo passa e a partícula desenvolve sua trajetória. 
É importante salientar que os observáveis intrínsecos também são invariantes. Contudo, eles são invariantes absolutos, números reais constantes que não dependem de características do movimento do sistema. Os invariantes dinâmicos, por outro lado, são observáveis extrínsecos que dependem, no geral, de outros observáveis do sistema, mas que se manterão invariantes em razão das propriedades do próprio movimento.

Antes de introduzir outros dois postulados com a implementação desta ideia, vamos tratar do primeiro e mais famoso exemplo de invariante dinâmico, a Energia.

\section{Energia}

Vamos começar a definir o observável energia através de duas propriedades:

1. A energia é uma quantidade conservada por rotações, portanto, é um observável escalar.

2. A energia é um observável aditivo.

Essas propriedades ainda não são suficientes, mas podemos começar por aqui. A primeira propriedade, a de que a energia é um escalar, nos permite determinar que a energia poderia ser um observável intrínseco da partícula. Contudo, vamos descartar esta possibilidade e assumir, por princípio, que a energia é um observável extrínseco, portanto, dependente do movimento. Assim, a energia de um sistema deve depender de observáveis euclidianos como o tempo, a posição, a velocidade e a aceleração. O tempo é um número real, mas os demais observáveis são vetores e, até aqui, a única maneira de produzir escalares a partir de vetores é através do produto escalar.

A aditividade da energia, por outro lado, nos permite supor que a energia total de um sistema possui várias origens que são somadas. A energia depende:

- Do movimento do sistema com relação a um determinado sistema referencial;

- Do movimento interno das partículas constituintes do sistema;

- Da interação do sistema com outros sistemas.

- Da interação entre as partículas internas do sistema.

Estas são as origens mecânicas da energia. Fora do escopo da mecânica clássica, precisamos falar de mais três tipos de energia:

- Energia intrínseca devido à existência da massa $\left(E=m c^{2}\right)$.

- Energia dos campos de interação.

- Energia interna termodinâmica.

A energia que vem do movimento do sistema físico com relação a um referencial é denominada energia cinética. Por outro lado, se o sistema físico é composto por muitas partículas, o movimento relativo entre as partículas é responsável por parte da energia total do sistema. Em parte, essa energia origina a energia interna termodinâmica. Contudo, o movimento interno das partículas não é a única origem da energia interna. Parte desta energia vem do calor, que tem origem estatística, mas essa discussão foge ao nosso escopo. Estamos interessados apenas nas energias de origem mecânica. Um sistema físico também pode interagir com outros sistemas físicos, assim, uma interação externa é uma fonte de energia total de um sistema. Ainda, se as partículas internas de um sistema interagem entre si, esta interação também dá origem a uma energia. As energia de interação são comumente denominadas energia potencial.

Sobre as formas não mecânicas da energia, temos o exemplo da energia intrínseca de uma partícula devido a sua massa. Provavelmente a equação mais famosa da física, $E=m c^{2}$ determina o conteúdo de energia da massa. Esta energia é usualmente ignorada nos processos mecânicos, químicos e biológicos em razão da conservação da massa. Portanto, a mecânica clássica, para a qual a massa é um observável intrínseco imutável, não prevê fenômenos nos quais um conteúdo de matéria pode ser convertido em energia. Ainda assim, tais fenômenos existem e constituem numerosos exemplos, como as bombas de fissão e fusão nuclear, e a produção de energia nas estrelas. 
Ademais, temos a energia devida à existência dos campos de interação, assunto marginal para a mecânica clássica, mas importante, por exemplo, no eletromagnetismo e nas demais descrições das interações fundamentais. A rigor, a mecânica clássica não introduz campos de interação, embora a gravitação universal newtoniana possa ser um exemplo controverso. Na eletrodinâmica, duas partículas carregadas formam um sistema que não só tem energia cinética e de interação mútua, mas que produzem campos elétricos e magnéticos também detentores de energia. Tal energia pode, inclusive, ser perdida mesmo que o sistema em si seja mecanicamente isolado, através de ondas eletromagnéticas.

\section{Mais dois postulados}

Para implementar as ideias acima, vamos introduzir mais dois postulados:

Postulado 5: (Princípio da Inércia - Primeira Lei de Newton). Existe uma classe especial de sistemas referenciais, denominados Referenciais Inerciais, para os quais a velocidade de uma partícula livre é constante.

Postulado 6: A energia total de um sistema isolado é preservada durante o seu movimento.

Não há como fugir da Primeira Lei de Newton. De fato, ela é mais importante para introduzir a classe de referenciais inerciais que para determinar o movimento da partícula livre. Como veremos, este movimento pode ser deduzido a partir do sexto postulado.

Os referenciais inerciais são aqueles, portanto, que medem a velocidade de uma partícula livre como um vetor constante, ou seja, de aceleração nula. Para encontrarmos um referencial inercial, basta encontrarmos uma partícula livre e nos colocarmos em movimento uniforme com relação a esta partícula. Uma vez que um referencial inercial é determinado, teremos infinitos referenciais inerciais, uma vez que dois referenciais inerciais se movem com velocidade constante com relação à partícula livre e, portanto, se moverão com velocidade mutualmente constante.

Portanto, uma vez escolhido um referencial inercial, a velocidade de uma partícula livre é um invariante dinâmico vetorial e a energia total de um sistema de partículas que não interage com outros sistemas externos é um invariante dinâmico escalar.

\section{A partícula livre}

Os seis postulados apresentados até aqui são suficientes para deduzir o movimento unidimensional, como o caso de uma partícula livre. Já que uma partícula livre move-se em linha reta com relação a um referencial inercial, basta que escolhamos um referencial inercial cuja orientação alinhe, por exemplo, o eixo $\mathbf{e}_{1}$ à trajetória. Uma vez que a partícula se movimenta apenas sobre este eixo, podemos ignorar os demais eixos, então, apenas uma coordenada é suficiente para determinar a trajetória $x=x(t)$ da partícula.

Obviamente, a primeira lei de Newton é suficiente para deduzir como a partícula livre se move sobre a reta. Se ela se move com velocidade constante $v$, isto implica em $\dot{x}=v$ e, assim, uma integração no tempo resulta na equação da reta $x=x_{0}+v t$. Aqui, o sinal de $v$ indica o sentido do movimento.

Contudo, é o postulado 6 que determina a propriedade dinâmica do movimento, uma vez que ele determina o invariante dinâmico relacionado à curva percorrida pela partícula. Notemos que, em primeiro lugar, a energia total de uma partícula não possui contribuição interna, uma vez que uma partícula não tem estrutura. Visto que a partícula é livre, também não há interação externa. Assim, a única contribuição para a energia de uma partícula livre é a energia cinética, se ignorarmos origens não mecânicas para a energia.

Portanto, a energia de uma partícula livre é um escalar euclidiano, invariante durante o movimento e que consiste apenas na energia cinética. Para que a energia seja um escalar, precisamos saber as expressões en- 
volvendo os observáveis extrínsecos que temos até então que resultam em escalares. Como nossos observáveis fundamentais são vetoriais, posição, velocidade e aceleração, temos que usar produtos escalares para contruir uma boa expressão para a energia. Os candidatos disponíveis são:

- $\mathbf{x}^{2}=\mathbf{x} \cdot \mathbf{x} ; \mathbf{x} \cdot \mathbf{v} ; \mathbf{x} \cdot \mathbf{a}$

- $\mathbf{v}^{2}=\mathbf{v} \cdot \mathbf{v} ; \mathbf{v} \cdot \mathbf{a}$

- $\mathbf{a}^{2}=\mathbf{a} \cdot \mathbf{a}$.

Contudo, como estamos lidando com uma partícula livre, a aceleração é nula. Assim, a energia da partícula deve ser uma combinação linear

$$
E_{c}=\alpha \mathbf{x}^{2}+\beta \mathbf{v}^{2}+\gamma \mathbf{x} \cdot \mathbf{v} .
$$

Agora, esta energia deve ser uma constante do movimento. Assim,

$$
\frac{d E_{c}}{d t}=0 \Longrightarrow \frac{d}{d t}\left(\alpha \mathbf{x}^{2}+\beta \mathbf{v}^{2}+\gamma \mathbf{x} \cdot \mathbf{v}\right)=0 .
$$

Como resultado, temos

$$
2 \alpha \mathbf{x} \cdot \mathbf{v}+2 \beta \mathbf{v} \cdot \mathbf{a}+\gamma\left(\mathbf{v}^{2}+\mathbf{x} \cdot \mathbf{a}\right)=0 .
$$

Tomando a aceleração nula, vemos que o coeficiente $\beta$ não precisa ser nulo. Contudo, a equação restante

$$
(2 \alpha \mathbf{x}+\gamma \mathbf{v}) \cdot \mathbf{v}=0
$$

é obedecida se $\alpha=0$ e $\gamma=0$. Poderíamos ter a solução $\mathbf{v}=-\frac{2 \alpha}{\gamma} \mathbf{x}$, contudo, esta não é uma opção para $\mathbf{v}$ constante ( $1^{a}$. Lei de Newton), a não ser que $\mathbf{x}$ seja constante, o que não queremos. Com $\alpha=\gamma=0$, a energia cinética tem a forma $E_{c}=\beta \mathbf{v}^{2}$, sobre a qual nos resta estabelecer o valor de $\beta$.

A costante $\beta$ só pode ser fixada empiricamente, através de experimentos. No caso da partícula livre, podemos fixa-lo analisando o movimento de bolas em um trilho horigontal. Encontraríamos que o valor da energia cinética de bolas lançadas com a mesma velocidade inicial seria proporcional à massa da bola. Vamos fixar o valor de $\beta$ em metade da massa da partícula, assim,

$$
E_{c}=\frac{1}{2} m \mathbf{v}^{2}
$$

Como $E_{c}$ é uma constante, um número real positivo, tratamos agora de uma partícula livre com energia $E_{c}=$ $E$ constante. Portanto, diferentes valores de $E$ para uma mesma partícula resultarão em diferentes curvas sobre a reta, ou seja, a partícula percorrerá sua trajetória com velocidades diferentes para energias diferentes. Note que, neste caso, temos

$$
\frac{1}{2} m v^{2}=E \Longrightarrow v^{2}=\frac{2 E}{m} \Longrightarrow v= \pm \sqrt{\frac{2 E}{m}} .
$$

Como a partícula tem uma trajetória reta, o módulo de sua velocidade depende da energia. O sinal \pm indica apenas que o sentido pode ser positivo ou negarivo no eixo. Isto também significa que

$$
\frac{d x}{d t}= \pm \sqrt{\frac{2 E}{m}} \Longrightarrow d x= \pm \sqrt{\frac{2 E}{m}} d t
$$

que é uma equação diferencial total. Integrando em ambos os lados, temos

$$
\int_{x_{0}}^{x} d x^{\prime}= \pm \sqrt{\frac{2 E}{m}} \int_{t_{0}}^{t} d t \Longrightarrow x-x_{0}= \pm \sqrt{\frac{2 E}{m}}\left(t-t_{0}\right) .
$$

Vamos acertar o relógio do observador para $t_{0}=0$. Assim,

$$
x=x_{0} \pm t \sqrt{\frac{2 E}{m}},
$$


que é a equação horária de uma partícula com velocidade $\pm \sqrt{2 E / m}$ constante sobre uma reta.

A partir daqui, a energia cinética será sempre dada pela expressão (??). Veremos nas próximas aulas que, para sistemas em uma dimensão, cuja trajetória é restrita a uma reta, os seis primeiros postulados são suficientes para descrever a dinâmica de partículas. Contudo, outros dois postulados serão necessários quando o sistema físico tiver total liberdade em três dimensões. 